\title{
Impacto do uso de fibras poliméricas no desempenho de concretos refratários aplicados por projeção
}

\section{(Influence of the polymeric fibers on shotcrete refractory castables)}

\author{
D. Vasques Filho, Y. A. Marques, R. G. Pileggi, V. C. Pandolfelli \\ Departamento de Engenharia de Materiais - DEMa \\ Universidade Federal de S. Carlos \\ Rod. Washington Luiz, km 235, C. P. 676, S. Carlos, SP, 13565-905 \\ demivasques@polvo.ufscar.br,vicpando@power.ufscar.br
}

\begin{abstract}
Resumo
A utilização de fibras poliméricas em concretos refratários tem sido motivada principalmente pela possibilidade de se elevar a velocidade de secagem sem acentuar o risco de explosão destes materiais. As fibras quando empregadas em concretos aplicados por projeção a úmido podem melhorar o ancoramento e reduzir a formação de trincas de secagem do material. Contudo, os benefícios que as mesmas exercem sobre a adesão do concreto na superfície precisam ainda ser investigados e quantificados, sendo estes os objetivos principais deste trabalho. Para tanto, foi utilizada a técnica de reometria de concretos para investigar a influência da adição de fibras de polipropileno, em tamanhos ( $3 \mathrm{~mm}$ e $6 \mathrm{~mm})$ e teores $(0,045 \%$ e $0,09 \%$ em peso) distintos, sobre o desempenho de projeção de uma composição bombeável de alta alumina. Os resultados demonstraram que o impacto das fibras em concretos de projeção depende da condição inicial do sistema onde são adicionadas.

Palavras-chave: concreto, fibras poliméricas, projeção, reômetro, refratários.
\end{abstract}

Abstract

The use of polymeric fibers in refractory castables has been motivated due to the possibility of improving the drying performance and reducing the explosion risks of these materials. For shotcrete castables, fiber addition can also promote bridging and avoid dryingcracks. However, the benefits resulting by fiber-containing castables on the surface adhesion still need to be evaluated and quantified. This was the main purpose of this work which was based on the castables reometry's technique in order to analyze the influence of polypropylene fibers of different sizes $(3 \mathrm{~mm}$ and $6 \mathrm{~mm})$ and amounts $(0.045 \%$ and $0.09 \%$ on weight) on the shotcrete performance of a pumpable high-alumina composition. The results showed that the fiber's benefits depend on the material initial characteristics.

Keywords: castables, polymeric fibers, shotcrete, rheometer, refractories.

\section{INTRODUÇÃO}

A técnica de projeção a úmido consiste em aplicar o concreto bombeado na forma de um jato de "spray" sobre a superfície a ser recoberta. Para tanto, na parte final da tubulação de bombeamento é acoplado um bocal de projeção que possui duas entradas, sendo uma para a injeção de um jato de ar comprimido que acelera o concreto para a formação do "spray" e a outra para a introdução do aditivo de projeção que é responsável pela perda instantânea da fluidez do concreto que atinge a superfície [1].

Esta técnica reduz o custo de instalação e permite que uma grande quantidade de material seja aplicada em um curto intervalo de tempo sem a necessidade de moldes [1]. Apesar das vantagens oferecidas pela projeção, apenas recentemente esta técnica ganhou espaço no setor de refratários, sobretudo devido aos avanços tecnológicos dos equipamentos de aplicação, às melhorias dos concretos bombeáveis e ao aperfeiçoamento dos aditivos de projeção (busca por aditivos orgânicos isentos de álcalis) [2].

Além desses avanços, é cada vez mais freqüente o uso de fibras poliméricas na projeção a úmido [2]. As fibras proporcionam um grande potencial de ancoramento do material aplicado, podendo reduzir a perda por rebote e/ou evitar a formação de trincas de retração durante o processo de secagem e endurecimento [3].

As fibras também permitem o aumento na velocidade de secagem destes materiais [4], diminuindo o risco de explosão e impedem a fragmentação do material consolidado quando camadas sucessivas de concreto são aplicadas.

Neste contexto, a utilização de fibras se torna um fator de desenvolvimento tecnológico dos concretos aplicados por projeção. Contudo, estudos sistemáticos sobre a adição e desempenho das fibras na projeção ainda são escassos. Desta forma, este trabalho tem como objetivo avaliar, através da técnica de reometria, a influência das fibras poliméricas (tamanho e teor) sobre o comportamento de projeção de concretos refratários de alta alumina preparados com diferentes teores de água. 


\section{MATERIAIS E MÉTODOS}

A composição do concreto (Tabela I) utilizado neste trabalho foi baseada no modelo de empacotamento de Andreasen com coeficiente de distribuição $q=0,26$, sendo as matérias-primas e o cimento, fornecidos pela Alcoa - Brasil. $\mathrm{O}$ dispersante utilizado foi um policarboxilato éter (SKW Alemanha) e o aditivo de projeção empregado foi uma combinação de $0,4 \%$ em peso de poliacrilato de sódio (BASF - Alemanha) de peso molecular de $15000 \mathrm{~g} / \mathrm{mol}$ e $0,025 \% \mathrm{em}$ peso de alginato de sódio (Fluka - Suíça).

Tabela I - Composição do concreto $(q=0,26)$ de alta alumina estudado.

[Table I - High-alumina castable composition $(q=0,26)$.]

\begin{tabular}{|c|c|c|c|}
\hline & $\begin{array}{l}\text { Matérias } \\
\text { Primas }\end{array}$ & $\begin{array}{c}\% \\
\text { volume }\end{array}$ & $\begin{array}{c}\% \\
\text { peso }\end{array}$ \\
\hline \multirow{4}{*}{$\begin{array}{l}\text { Matriz } \\
(<100 \mathrm{Pm})\end{array}$} & Aluminas & 17,0 & 17,4 \\
\hline & Calcinadas & & \\
\hline & Cimento CA 14 & 2,0 & 1,6 \\
\hline & AEB 200/F & 16,0 & 16,0 \\
\hline $\begin{array}{l}\text { Agregado } \\
(<100 \mathrm{Pm})\end{array}$ & AEB & 65,0 & 65,0 \\
\hline
\end{tabular}

AEB: Alumina Eletrofundida Branca, Aluminas Calcinadas = A1000 SG e A3000 FL; CA 14 = Cimento de Aluminato de Cálcio. [AEB: White Fused Alumina, Calcined Alumina = A1000 SG e A3000 FL; CA 14 = Calcium Aluminate Cement.]

Fibras de polipropileno foram testadas em tamanhos de 3 $\mathrm{mm}$ e $6 \mathrm{~mm}$, nos teores de $0,045 \%$ e $0,09 \%$ em peso $(0,18 \%$ e $0,36 \%$ em volume) do concreto. As fibras foram adicionadas durante a fase de mistura a seco do material e o concreto foi preparado com $16 \%$ e $18 \%$ vol. de água.

As composições foram misturadas em um reômetro para concretos [5] a uma rotação de $33 \mathrm{rpm}$, registrando-se o torque de resposta do material, de acordo com o procedimento descrito a seguir:

a) homogeneização do concreto a seco durante $30 \mathrm{~s}$; $150 \mathrm{~s}$;

b) adição de água necessária para o início da virada por

c) adição do restante de água e homogeneização por um tempo adicional de $60 \mathrm{~s}$.

Para a execução do estudo foi utilizada uma seqüência experimental desenvolvida para a análise de materiais de projeção [6] de acordo com as etapas do processo. Diferentes ensaios simularam o bombeamento, a formação do "spray", adesão e consolidação do material na superfície:

(1) Ciclos de Cisalhamento: o material foi misturado a 33 rpm e então submetido a diferentes taxas de cisalhamento ( 0 a $75 \mathrm{rpm})$. Este ensaio indica as características reológicas do concreto, como a sua susceptibilidade ao bombeamento.

(2) Ensaio de Adesão: após a mistura do concreto, o aditivo de projeção foi injetado no mesmo instante em que a rotação foi elevada a $75 \mathrm{rpm}$, simulando o alto cisalhamento no bocal da projeção para a formação do "spray". A alta rotação foi mantida por $10 \mathrm{~s}$, sendo então reduzida a zero. A projeção neste ensaio é feita em um dispositivo cônico revestido com uma tela de fixação e com parede rugosa, o qual foi inserido no interior da cuba de mistura [6]. A porcentagem de massa não aderida ao dispositivo determina a perda por rebote.

(3) Perfil de Endurecimento: após a mistura e homogeneização do material, o aditivo de projeção foi injetado ao mesmo tempo em que a rotação foi elevada a $75 \mathrm{rpm}$ por $10 \mathrm{~s}$. A rotação foi então reduzida para $20 \mathrm{rpm}$ por $70 \mathrm{~s}$. As etapas deste ensaio simulam a formação do "spray" (alto cisalhamento) e a consolidação do material na superfície de aplicação. A representação esquemática da sequiência experimental adotada neste ensaio e os parâmetros obtidos do mesmo são apresentados na Fig. 1.
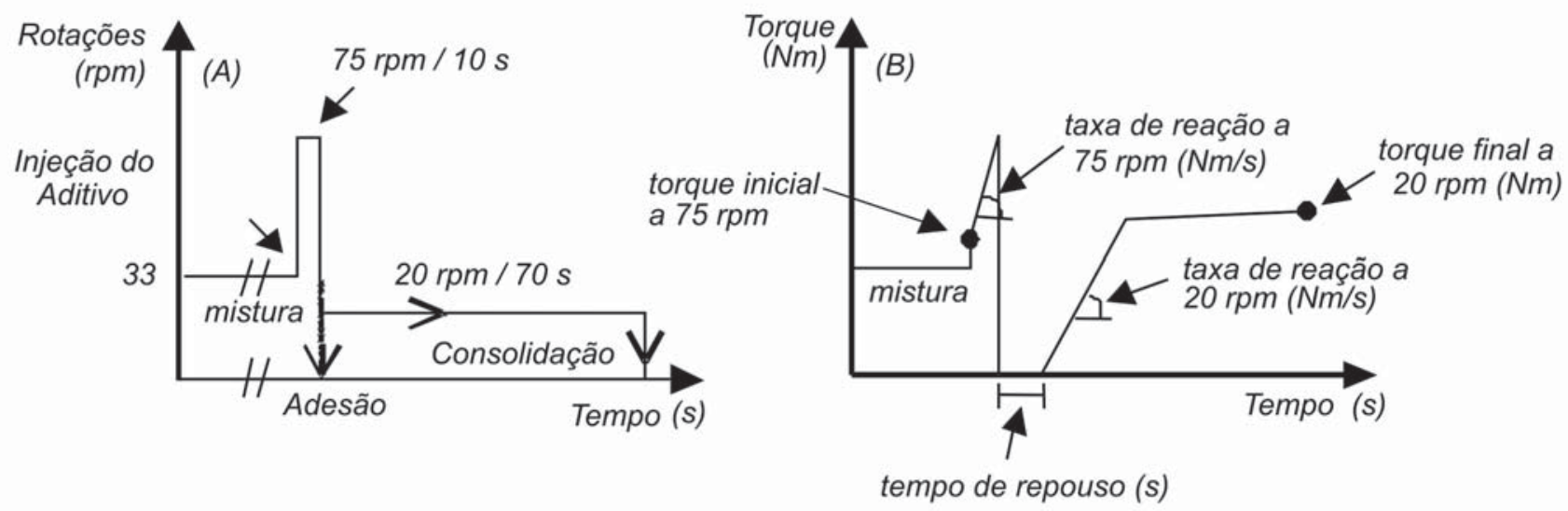

Figura 1: (a) Seqüência de ensaio para os testes de adesão e perfil de endurecimento; (b) variação de torque no ensaio de perfil de endurecimento e os parâmetros de projeção estimados.

[Figure 1: (a) Revolution speed set-up for sticking test and consolidation test; (b) torque variation for the consolidation test and the shotcrete parameters evaluated.] 


\section{PROJEÇÃO A ÚMIDO DE CONCRETOS REFRATÁRIOS}

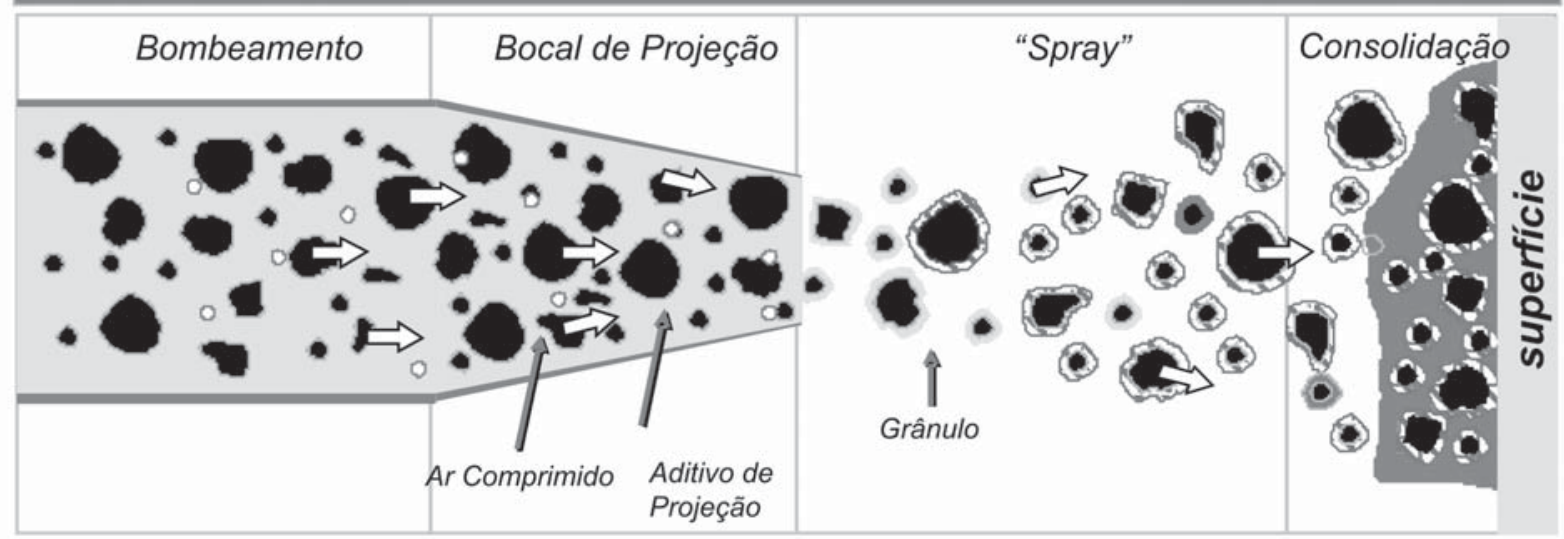

agregado

( matriz modificada pelo aditivo

bolhas de ar

matriz fluida

material endurecido

Figura 2: Ilustração esquemática da aplicação de concretos refratários por projeção: (a) bombeamento; (b) bocal de projeção; (c) "spray" e (d) consolidação.

[Figure 2: Schematic illustration for shotcrete of castables: (a) pumping; (b) nozzle; (c) spray; (d) consolidation.]

\section{RESULTADOS E DISCUSSÃO}

A aplicação de concretos refratários pela técnica de projeção a úmido é um processo complexo que envolve, além da projeção propriamente dita, a mistura e o bombeamento do concreto [6]. A projeção em si constitui apenas a fase final deste processo sendo fundamental conhecer os fatores que influenciam as demais etapas. A Fig. 2 ilustra os fenômenos envolvidos na projeção a úmido a partir do bombeamento.

\section{(A) Mistura}

A etapa da mistura influi diretamente nas características reológicas do concreto, sobretudo com a introdução de fibras no material. A solicitação mecânica nesta etapa é capaz de degradar as fibras de maneira proporcional à energia dispensada durante o processo [7]. Na Fig. 3 são apresentadas as curvas de torque na mistura dos sistemas estudados e os valores de esforço de mistura calculados em cada caso.

Com $16 \%$ vol. de água, o concreto sem fibras teve seu tempo de virada próximo ao tempo estipulado para a mistura (240 s). A adição de fibras reduziu o tempo de virada permitindo maior eficiência neste processo. Por este motivo, todos os sistemas tiveram valores de esforço de mistura próximos daquele da mistura do concreto de referência (sem fibras). A exceção foi o sistema com maior tamanho e teor de fibras $(6 \mathrm{~mm} / 0,09 \%)$ o qual sofreu maior solicitação e, provavelmente superior degradação das fibras devido ao maior atrito gerado pela maior coesão e ancoramento neste sistema.

Com 18\% vol. de água os sistemas apresentaram menores diferenças entre os esforços de mistura. Vale ressaltar que apesar dos valores de esforço serem próximos (com exceção do sistema $6 \mathrm{~mm} / 0,09 \%$ onde a diferença foi perceptível), as fibras de $6 \mathrm{~mm}$ provavelmente apresentam tamanho médio final menor do que o tamanho nominal por se degradarem
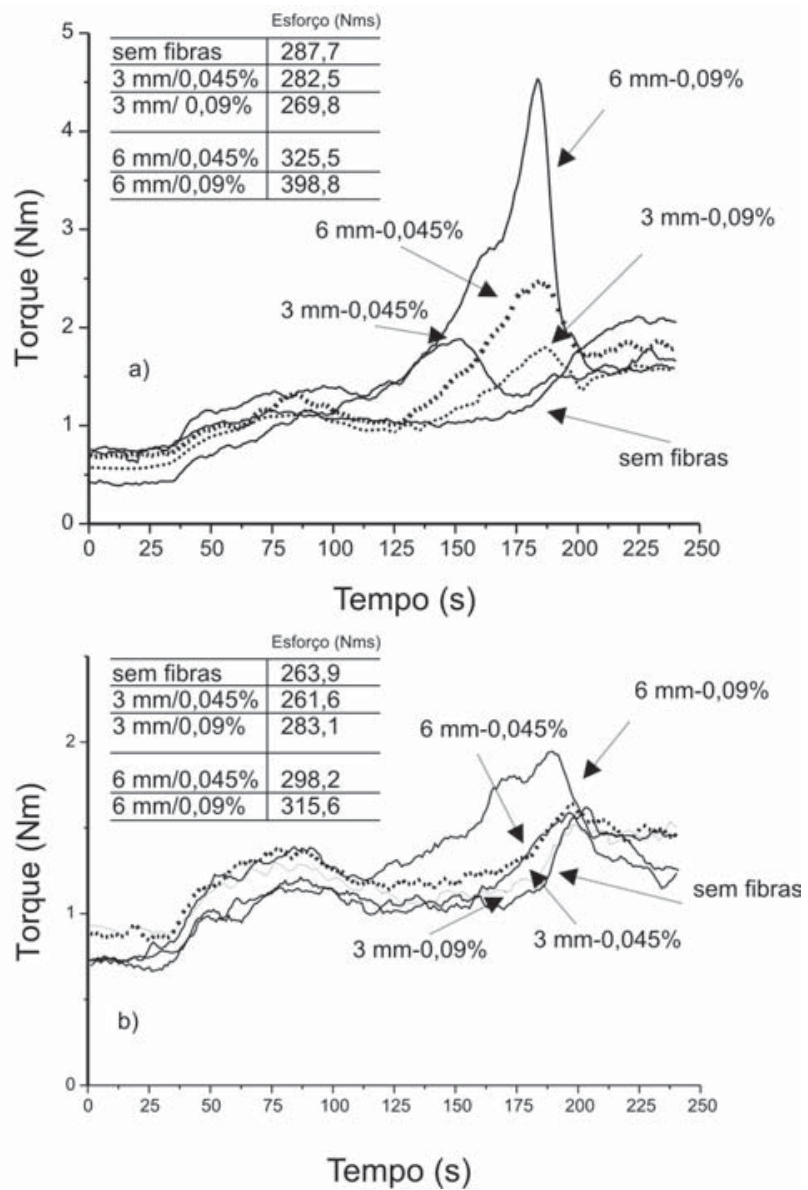

Figura 3: Curvas de mistura para (a) $16 \%$ e (b) $18 \%$ vol. de água. Valores de esforço de mistura estão apresentados nas tabelas auxiliares.

[Figure 3: Mixing curve for (a) 16\% and (b) 18\% vol. water. Mixing effort values are presented in the enclosed tables.] 
(proporcionalmente ao esforço de mistura) mais que as fibras de $3 \mathrm{~mm}$, que praticamente mantêm o tamanho inicial segundo a literatura [7].

\section{(B) Bombeamento}

Para que a projeção ocorra de maneira adequada, o concreto deve inicialmente apresentar comportamento bombeável e natureza pseudoplástica. Além disso, o mesmo deve apresentarse homogêneo, disperso, isento de segregação e com baixa viscosidade para facilitar a formação do "spray" na saída do bocal de projeção [6].
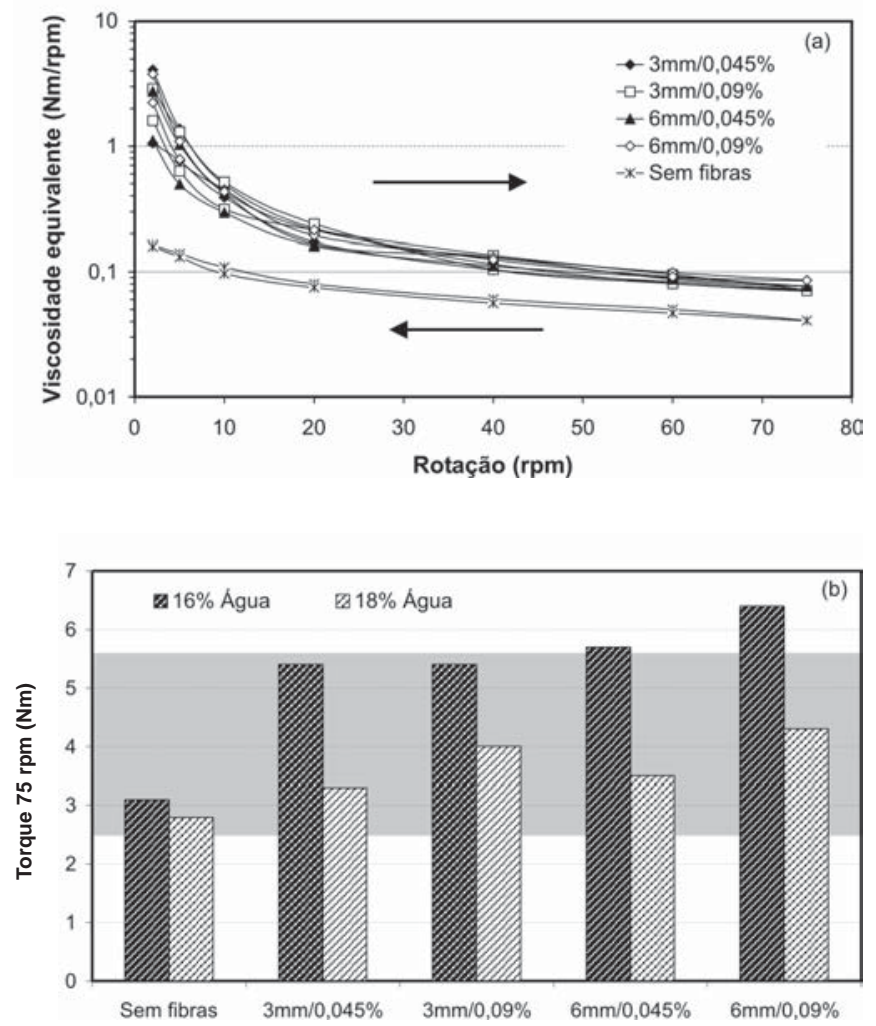

Figura 4: (a) Viscosidade equivalente para os sistemas com $16 \%$ vol. de água. As flechas indicam que os níveis de torque são menores no ciclo de ida, exceto para o concreto puro. (b) Valores de torque a $75 \mathrm{rpm}$. A região recomendada para concretos bombeáveis está destacada na área mais escura da figura.

[Figure 4: (a) Equivalent viscosity for 16\% vol. water systems. Arrows indicate that torque levels are lower at the beginning of cycle than at the end, except for pure castable. (b)Torque values at 75 rpm. Dark region indicates the torque suitable for pumpable castables.]

A Fig. 4a mostra a viscosidade equivalente (neste trabalho calculada pela razão torque/rotação do ciclo de cisalhamento) para os sistemas com $16 \%$ vol. de água que são os mais críticos pela menor quantidade de líquido. As composições apresentaram características pseudoplásticas, porém a adição de fibras dificultou o escoamento tanto para baixas quanto para altas taxas de cisalhamento.
As fibras acentuaram a pseudoplasticidade das composições, como observado pelo aumento da inflexão das curvas na Fig. 4a. Este comportamento é explicado pelo fato de que em baixas rotações, a viscosidade estimada dos concretos com fibras é bem maior do que a apresentada pelo concreto referência, devido ao superior grau de coesão das partículas. Entretanto essa viscosidade é reduzida em altas rotações chegando a valores similares, devido ao alinhamento das fibras em relação às linhas de fluxo do material.

No trecho de volta do ciclo (redução de rotação), os níveis de torque e a viscosidade equivalente foram superiores que no trecho de ida (aumento da rotação) nos sistemas com fibras. Este comportamento é característico de início de pega do concreto devido ao atrito e conseqüente aumento de temperatura gerado pela coesão acentuada com a adição de fibras.

Ainda para 16\% vol. de água, de acordo com a Fig. 4b, todas as composições apresentaram-se bombeáveis, exceto os sistemas contendo fibras de $6 \mathrm{~mm}$. Nestas composições os níveis de torque a $75 \mathrm{rpm}$ excederam a região indicada para o bombeamento [5].

Com $18 \%$ vol. de água o comportamento da viscosidade equivalente dos sistemas é semelhante, porém com menores níveis de torque. Com esta redução, todos os sistemas se caracterizaram como bombeáveis, no entanto com maiores riscos de segregação [5].

\section{(C) Projeção}

A perda de material por rebote (material não aderido na superfície projetada) na Fig. 5, apresentou valores bastante distintos entre os sistemas estudados. O sistema 16\% vol. de água/sem fibras foi utilizado como referência. A formação do "spray", a coesão do concreto e a taxa de reação determinada pelo aditivo apresentaram-se adequados proporcionando o bom desempenho relativo deste sistema.

Em aplicações industriais os resultados de rebote tenderiam a ser inferiores, devido às condições favoráveis de projeção encontradas nestes casos, como por exemplo, a maior distância e constância do ângulo de aplicação.

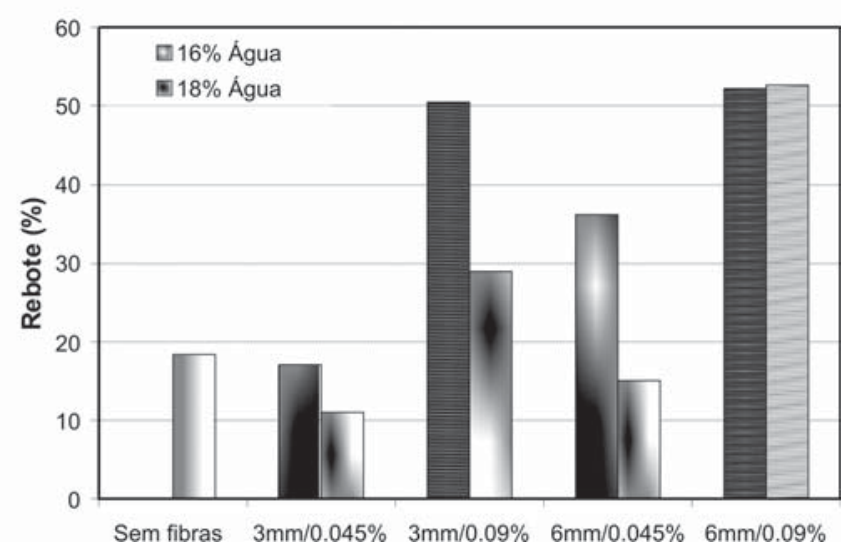

Figura 5: Material não aderido (rebote) para todos os sistemas testados.

[Figure 5: Material rebound for each studied system.] 
Com $16 \%$ vol. de água, a adição de fibras não conseguiu manter a adesão no nível obtido pelo concreto referência. Todos os sistemas com este teor de água prejudicaram o desempenho da projeção seguindo uma tendência do torque a $75 \mathrm{rpm}$ que é um indicativo da coesão do sistema. Desta forma, os sistemas mais coesos dificultaram a formação do spray resultando em uma maior perda de material.

Além disso, o teor de fibras causa maior impacto na perda por rebote do concreto do que o tamanho das mesmas. No fim do processo de projeção, o sistema $3 \mathrm{~mm} / 0,09 \%$ apresentou maior dificuldade em formar o "spray" do que o sistema 6 $\mathrm{mm} / 0,045 \%$, ou seja, aumentando o teor de fibras (de $3 \mathrm{~mm} /$ $0,045 \%$ para $3 \mathrm{~mm} / 0,09 \%$ ) o sistema sofreu maior influência do que com o aumento do tamanho. Tal fato pode estar relacionado à quebra das fibras, sobretudo as de $6 \mathrm{~mm}$, após a intensa solicitação mecânica na mistura e a altas taxas de cisalhamento.

Ao aumentar o teor de água a coesão do sistema diminuiu. Para os sistemas com $18 \%$ vol. de água, o concreto sem fibras obteve rebote relativamente alto quando comparado ao de referência com $16 \%$. Neste caso a quantidade de aditivo utilizada não foi suficiente para a quantidade de água e a perda de material foi causada pelo escoamento por fluência do concreto na superfície [6].

Adicionalmente, com $18 \%$ de água, a presença das fibras também seguiu a mesma tendência dos níveis de torque a 75 rpm para o rebote de cada sistema. Entretanto, as fibras de 3 $\mathrm{mm}$ e $6 \mathrm{~mm}$ para o teor de $0,045 \%$ em peso, tiveram seu rebote reduzido em relação ao concreto com $16 \%$ vol. de água.

As fibras no concreto com maior teor de água, estão menos degradadas a partir do processo de mistura e formação do "spray" e por isso atuam mais eficientemente no ancoramento. Neste caso, as fibras aumentam a viscosidade da matriz para um valor próximo do desejado, impedindo o material de ceder por fluência ao aderir na superfície.

Desta forma em alguns sistemas a adição de fibras compensa a baixa concentração do aditivo de projeção e ajuda na otimização da coesão do material bem como na formação do "spray" e adesão.

O sistema $6 \mathrm{~mm} / 0,09 \%$, ainda com $18 \%$ de água, apresentou rebote inesperadamente alto. Provavelmente, a maior facilidade de mistura não causou a degradação de forma intensa das fibras que mantiveram, em grande parte, seu tamanho original. Com isso, pelo alto tamanho e teor utilizados,
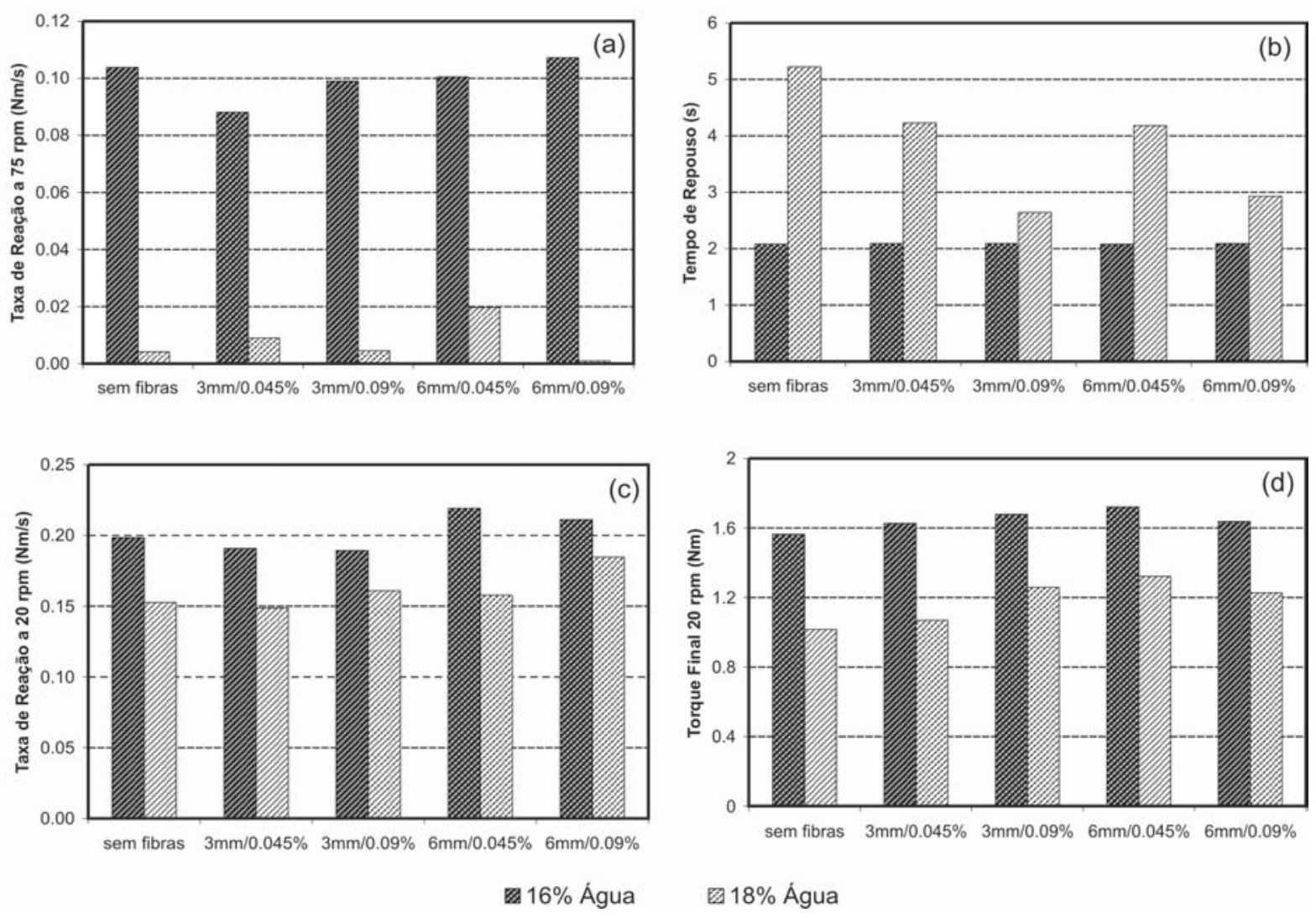

Figura 6: Parâmetros do ensaio de perfil de endurecimento. (a) taxa de reação a $75 \mathrm{rpm}$; (b) tempo de repouso; (c) taxa de reação a $20 \mathrm{rpm}$, indicando a consolidação do material e (d) torque final a $20 \mathrm{rpm}$ que representa a resistência adquirida pelo concreto.

[Figure 6: Consolidation test's parameters. (a) reaction rate at $75 \mathrm{rpm} ;($ b) resting time; (c) reaction rate at 20 rpm that indicates the material's consolidation and (d) final torque at 20 rpm representing the castables resistance acquired on the surface.] 
facilita-se o seu emaranhamento, o que acaba por prejudicar a formação do "spray" e a homogeneização do concreto durante a projeção. Esse fenômeno é conhecido como "golf-ball" [6] e acarreta no aumento da perda de material por rebote.

Em paralelo, de acordo com o ensaio de perfil de endurecimento, as taxas de reação (Fig. 6a e 6c) mantiveramse bem próximas, em todos os casos, para um mesmo teor de água. Estes resultados indicam que as fibras não influem na taxa de reação do concreto e que o teor de água influenciou mais intensamente nestes valores. Além disso, as fibras poliméricas não modificam a consolidação do material na superfície não tendo papel fundamental quanto ao endurecimento do concreto (Fig. 6c).

A Fig. 6b mostra que para os casos de maior coesão de material, o tempo de repouso foi mais curto, ou seja, a perda de fluidez é mais rápida e a viscosidade da matriz aumenta antes mesmo do material chegar à superfície. Um valor alto de viscosidade prejudica a capacidade de deformação do concreto e proporciona maior rebote.

Finalmente, o torque final atingido a $20 \mathrm{rpm}$ (Fig. 6b) para cada caso, não apresenta maiores diferenças indicando que a resistência final obtida na superfície de aplicação foi independente da adição de fibras no concreto e, mais uma vez, depende apenas do aditivo de projeção e do teor de água.

\section{CONCLUSÕES}

Os concretos com $16 \%$ de água necessitam de maior esforço de mistura e sofrem maior solicitação do que os sistemas com $18 \%$, que apresentam condições de misturas brandas pela maior quantidade de água presente. Com isso as fibras do sistema $16 \%$ se degradam mais, sobretudo as de $6 \mathrm{~mm}$.

Em baixas rotações os sistemas com fibras apresentam alta viscosidade, necessitando maior pressão da bomba de concretos no início do bombeamento. Entretanto, a continuidade do processo é facilitada, pois as fibras se alinham às linhas de fluxo do material em altas taxas de cisalhamento.

Todos os casos apresentaram-se com características pseudoplásticas, porém os sistemas com fibras de $6 \mathrm{~mm}$ em ambos os teores para $16 \%$ de água não se caracterizaram como bombeáveis.
A adição de fibras não proporcionou uma adesão total do material na superfície (rebote zero). Para 16\% vol. de água as fibras apenas pioraram o desempenho de adesão do material. Porém, com $18 \%$, os concretos com fibras $3 \mathrm{~mm} / 0,045 \%$ e 6 $\mathrm{mm} / 0,045 \%$ tiveram o rebote diminuído. Neste caso, a presença das fibras poliméricas compensou a baixa concentração do aditivo de projeção, reduzindo a perda por escoamento, causa do rebote relativamente alto do concreto sem fibras.

\section{AGRADECIMENTOS}

Os autores agradecem à FAPESP e ao CNPq, bem como a ALCOA-Alumínio S.A. pelo apoio dado à realização deste trabalho.

\section{REFERÊNCIAS}

[1] R. G. Pileggi, Y. A. Marques, D. Vasques Filho, A. R. Studart; V. C. Pandolfelli, "Wet-Shotcrete Additives", Am. Ceram. Soc. Bull. 81, 10 (2002) 51-57.

[2] L. I. Glassgol; "Refractory Shotcrete - The Current State of the Art"; Shotcrete; Summer (2002) 24-32.

[3] W. Sun, H. Chen, X. Luo, H. Qian, "The effect of hybrid fiber and expansive agent on the shrinkage and permeability of high-performance concrete", Cement and Concrete Research 31 (2001) 595-601.

[4] M. D. M. Innocentini, R. Salomão, C. Ribeiro, F. A. Cardoso, L. R. M. Bittencourt, R. P. Rettore, V. C. Pandolfelli, Am. Ceram. Soc. Bull. 81, 6 (2002) 34-37.

[5] R. G. Pileggi, V. C. Pandolfelli, "Rheology and particle size distribution of pumpable refractory castables", Am. Ceram. Soc. Bull. 80, 10 (2001) 52-57.

[6] R. G. Pileggi, Y A. Marques, D. Vasques Filho, V. C. Pandolfelli, "Shotcrete Performance of Refractory Castables", Refractories Applications 8, 3 (2003) 15-20.

[7] R. Salomão, C. S. Isaac, V. G. Domiciano, R. G. Pileggi, M. D. M. Innocentini, V. C. Pandolfelli, L. R. M. Bittencourt, "Influência da Mistura na Permeabilidade de Concretos Refratários Contendo Fibras Poliméricas", Anais do Congresso Brasileiro de Engenharia e Ciências dos Materiais (2002).

(Rec.04/06/03, Ac. 15/07/03) 